\title{
Classification of the intracranial hypertension and characteristics of intracranial pressure increase. Review
}

\author{
St.M. Iencean ${ }^{1}$, D. Adam ${ }^{2}$ \\ ${ }^{1}$ Neurosurgery, "Grigore T. Popa" University of Medicine and Pharmacy Iasi \\ ${ }^{2}$ Neurosurgery, "Carol Davila" University of Medicine and Pharmacy, Bucharest
}

Intracranial hypertension represents the increase in the intracranial pressure (ICP) due to disorders of the regulating mechanisms for intracranial pressure. It is caused by the intrinsic changes in the volumes of the intracranial compartments: brain parenchyma, cerebrospinal fluid and blood content, and/or by adding a pathological volume (extrinsic - tumor, haematoma, cerebral abscess, etc.).

At first, the increase in the intracranial pressure is caused by the modification of the volume-pressure relations in correlation with time (the speed of occurrence and the duration of changes), which is induced by various etiologies; afterwards, the intracranial hypertension generates physiopathological disorders which cause specific modifications to the endocranial structures, and, therefore, the subsequent ICP increase is accompanied by its own symptomatology.

In relation to its causal mechanism, the intracranial hypertension is and it may remain a symptom (increased intracranial pressure), or it evolves as a syndrome (increased intracranial pressure associated with other signs and symptoms). There are intracranial pressure values that are considered to be at the normal limit or moderately increased values, which do not induce evolutionary physio-pathological mechanisms by themselves. The subsequent evolution of the intracranial hypertension as a syndrome results from the very transformation of the initial ICP increase into a pathogenic mechanism and from the progression of the characteristic symptoms, whose intensity may exceed even the symptomatology of the initial illness.

Depending on the etiology and on the pathogenic mechanism that generates the disorder in the volume-intracranial pressure relation, and based on the variation of the intracranial pressure in time, there is now an etiopathogenic classification of the intracranial hypertension, which includes both the etiopathogenic mechanisms and the clinical data (Iencean 2001, revised in 2003). Many of the previous observations and findings of other authors have foreseen this systematization.

\section{Parenchymatous intracranial hypertension}

It occurs in the expanding intracranial processes (cerebral tumors, intracranial haematoma, cerebral abscesses, etc.), in the traumatic cerebral edema, in the cerebral ischemia with hypotoxic cerebral edema, in general intoxications with neurotoxins (endogenous or exogenous), etc. The primary cerebral etiology is known and it generates modifications of the intracranial volume; the cerebral edema occurs afterwards, and it evolves in parallel to the increase in the ICP. The parenchymatous lesion initially occurs as a result of the 
intrinsic cerebral etiology and of the primary modifications in the intracranial volume (expansive, compressive, hypotoxic or traumatic cerebral edema). The parenchymatous intracranial hypertension may have a complete evolution to its acute form with a brain stem ischemia or cerebral hernia.

\section{Intracranial hypertension due to disorders of the cerebrospinal fluid dynamics}

The dynamics of the cerebrospinal fluid includes the circulation of the cerebrospinal fluid at the time of its creation at the level of the choroid plexuses, and until its passage into the venous circulation.

The dynamic disorders of the cerebrospinal fluid may be:

- circulation disorders of the cerebrospinal fluid from its creation until resorption, and

- disorders in the passage of the cerebrospinal fluid in the venous drainage system (resorption).

The circulation disorders of the cerebrospinal fluid are produced when there is an obstacle in the fluid itinerary (ventricular system, magna cistern, basal cisterns) due to the existence of a ventricular or para-ventricular tumor, intraventricular hemorrhage, or obstruction of various causes of the Sylvian aqueduct. A dilatation of the segments of the ventricular system occurs, those that are supra-adjacent to the obstruction, while the clinical chart and the evolution are related to the obstructive internal hydrocephaly.

The resorption disorders of the cerebrospinal fluid occur on account of the lesions of the anatomic structures that provide the passage of the cerebrospinal fluid from the sub-arachnoid spaces into the venous drainage system in acute meningitis, in sub-arachnoid hemorrhage, in meningitis carcinomatosis, in sarcoidosis chronic meningitis, etc. A thickening of the leptomeninx occurs, blocking the Pachioni arachnoid corpuscles, and decreasing the absorption of the cerebrospinal fluid. The cerebrospinal fluid accumulates in the subarachnoid spaces and in the ventricular system, leading to a communicating hydrocephaly, with a peri-ventricular hydrocephalic cerebral edema and with an intracranial hypertension syndrome, which is usually a sub-acute one (called the meninx intracranial hypertension). The clinical chart and the increase in the intracranial pressure resemble the intracranial hypertension syndrome in cases of cerebrospinal fluid circulation obstruction.

\section{Vascular intracranial hypertension}

The ICP increase is caused by disorders of the sanguine, cerebral or extra-cerebral circulation. The increased volume of the cerebral parenchyma in vascular $\mathrm{ICH}$ is produced by a cerebral edema or by an increase in the cerebral sanguine volume ("congestive brain"). The cerebral edema and/or the congestive cerebral parenchyma will lead to an increased intracranial pressure. The increase in the cerebral sanguine volume occurs when there is an increased intracranial arterial sanguine supply or a decreased blockage of the venous drainage. When the venous drainage is reduced, there is also a decrease in the resorption of the cerebrospinal fluid. The "congestive brain" aspect is caused by the increase in the intra-cerebral sanguine volume, and the volume-intracranial pressure conflict occurs. At the level of the structure represented by the nervous parenchyma - vascular capillary, two types of modifications are produced, which may be separate or have an evolutionary 
connection between them: the stage of a cerebral edema, due to water accumulation in the parenchyma, and the stage of congestive cerebral edematization (congestive brain), with an increase in the volume of the cerebral parenchyma due to vascular dilatation.

The vascular intracranial hypertension occurs in:

- cerebral illnesses: by affecting the venous return and blocking the cerebrospinal fluid absorption in cerebral venous thromboses, thrombosis of superior sagittal sinus, cerebral thrombophlebites, mastoiditis ("otitic hydrocephalus", described by Symonds - thrombosis of venous sinuses, secondary to otomastoiditis), etc., by slowing down or decreasing the venous flow in the superior longitudinal sinus in the arterio-venous shunt in certain types of intra-cerebral vascular malformations, especially Galien vein aneurysms, or by decreasing the sanguine feeding in cerebral ischemias with secondary ischemic cerebral edema, as in the ischemic stroke caused by the occlusion or stenosis of the big cerebral vessels, with a serious form of malign ischemic infarct within the territory of the middle cerebral artery (massive Sylvian infarct).

- extra-cerebral illnesses: hypertensive encephalopathy with congestive cerebral edematization and with extra-cellular hydrostatic cerebral edema (by ultrafiltration); in the cervical or thoracic venous blockage.

The vascular intracranial hypertension usually develops only until the occurrence of a complete ICH syndrome; but, sometimes, it may develop until the occurrence of the acute form of ICH due to the secondary disorders of the cerebral sanguine circulation. In the case of the massive ischemic infarct within the territory of the middle cerebral artery, the cerebral edema occurs quickly, it is extensive and the intracranial hypertension has an acute evolution, with a mortality of up to $80 \%$ of the cases.

\section{Idiopathic intracranial hypertension}

This form of ICH has also been designated as pseudotumor cerebri, essential or cryptogenic ICH, a terminology that is outdated. The etiology could not be established. It occurs in endocrine illnesses, metabolic disorders or various hematological illnesses, etc. Not long ago, physio-pathological mechanisms were thought to include various disorders of the cerebrospinal fluid secretion and absorption, so that the increase in the ICP is secondary to the decrease in the cerebrospinal fluid absorption without the occurrence of hydrocephaly (this theory is also sustained by the efficiency of the lumbar-peritoneal shunt). Moreover, the cerebral edema could be generated by the so-called non-specific "associated factors", which lead to the ICP increase, without the possibility of establishing an etiological relation.

The most probable current pathogenic hypothesis about the idiopathic intracranial hypertension is based on the dynamics of the intracranial fluid circuits that allow maintaining the auto-regulation of the cerebral circulation and of the cerebral sanguine flow. In idiopathic $\mathrm{ICH}$, there are various pathologic conditions that cause progressive damage to the hematoencephalic barrier with a hyper-production of interstitial fluid and the occurrence of the extra-cellular cerebral edema. The cerebrospinal fluid is normally produced, and the increased pressure from the cerebral parenchyma is equalized by the 
pressure of the cerebrospinal fluid by means of increased exchanges from the interstitial fluid towards the cerebrospinal fluid at the pial trans-cerebral and transependyma level. The increase in the fluid pressure is followed by the increase in the resorption of the cerebrospinal fluid and by a rapid venous efflux, so that the increased intracranial pressure does not affect the cerebral circulation, which is maintained within the normal limits. The transependyma and the trans-pial circuit of the interstitial fluid towards the cerebrospinal fluid represents and efficient compensating mechanism when the intracranial pressure increases gradually and allows the cerebral circulatory auto-regulation.

Therefore, the idiopathic ICH occurs due to the impact on the hematoencephalic barrier, by various causes that are not established yet, with a hyperproduction of interstitial fluid and an extracellular cerebral edema, with increased exchanges from the interstitial fluid towards the cerebrospinal fluid at the transependyma and pial trans-cerebral level; an increased resorption of the cerebrospinal fluid occurs, and there is a rapid venous efflux.

The idiopathic ICH develops into an incomplete ICH syndrome, despite the increased ICP values and the presence of the papillary edema.

The causes established for certain forms of ICH, which are considered idiopathic, will probably include them into one of the $\mathrm{ICH}$ forms with a known etiology: vascular, meningeal or parenchymatous.

The ICH syndrome may also occur as a form of transition between the four forms of ICH, or it may be induced by several concomitant pathogenic mechanisms of the cerebral edema in: bacillary meningoencephalitis, neoplasia, hydro- electrolytic disorders, diabetic keto-acidosis, PRES, etc.

The comparison between the ICH forms underlines the existing differences very clearly

The decompensation in parenchymatous intracranial hypertension is more rapid and it is produced at lower values than the ones in idiopathic ICH due to the existence of the pressure differences between the cranio-spinal compartments and the occurrence of the cerebral circulation disorders with a decrease in the cerebral perfusion pressure in parenchymatous ICH.

The increased critical values of the intracranial pressure, the period of ICP increase and the duration of the ICP action are the main parameters that control the intracranial biomechanical fluid stability, and which cause the pressure and circulatory decompensation. The state of clinical instability is produced by the passage to more increased values of the intracranial pressure, and it occurs when the ICP action time is longer than the compensation cerebral capacity.

The pressure-time fluctuation defines the pressure increase in relation to the action duration of the increased intracranial pressure, which induces the auto-regulation disorder of the cerebral sanguine circulation. The limit decompensation conditions occur when the duration of the increased ICP action is longer than the period corresponding to the same pressure values in the pressure compensation situation, or the ICP values are higher than the ICP values corresponding to the same duration of action.

The evolution of intracranial hypertension has three stages and it overcomes the critical ICP moments the moment it passes to the next stage: 
a. at first, the ICP increase is an alarm signal;

b. afterwards, the ICP increase exceeds the critical value for the occurrence of the $\mathrm{ICH}$ syndrome; the ICH syndrome evolves based on the existing etiology and the maintenance of auto-regulation of the cerebral sanguine circulation. The compensating mechanisms may stop the pressure-time fluctuation.

c. finally, the acute critical ICP values lead to the alteration of the circulatory autoregulation, and the compensating mechanisms may no longer stop the evolution towards the acute stage.

The first and the second stages are reversible, and the third stage corresponds to a phase of maximum instability when the decompensation with clinical aggravation occurs via brainstem ischemia or cerebral hernia, which generates irreversible cerebral lesions.

The etiological temporal relation classifies the causes of the intracranial hypertension into:

- acute causes that lead to the acute increase in the intracranial pressure and the rapid installation of the $\mathrm{ICH}$ syndrome, and which include the cranial-cerebral traumatisms, acute ischemic lesions or intra-cerebral hemorrhage; exogenous or endogenous intoxications with the rapid formation of the cerebral edema, as well as infections such as encephalitis or meningitis may lead to ICH, too;

- chronic causes, which produce the progressive increase in the intracranial pressure and the belated decompensation of the ICH; they include many of the intracranial tumors with slow evolution, progressive blockage of the cerebrospinal fluid circulation, the chronic subdural haematoma, the idiopathic intracranial hypertension, etc.

\section{Characteristics of intracranial pressure increase}

Intracranial pressure increase is specific for every pathogenic mechanism of cerebral illnesses, and the ICP monitoring reveals characteristic features that establish the clinical evolution of the intracranial hypertension:

- the intracranial pressure increasing speed to the normal limit values of $20 \mathrm{~mm}$ $\mathrm{Hg}$ and above this value: therefore, there are two segments of the ICP increase:

1. pressure increase segment up to the normal limit

2. pathological pressure increase segment, up to the maximum value

- the maximum pathological value reached by the intracranial pressure,

- duration of the pathological intracranial pressure maintenance,

- the period of recovery to normal pressure values

- the frequency of pressure increase reoccurrence or the frequency of remissions.

Several types of pressure increases may be described based on the above-mentioned characteristics and on the clinical evolution:

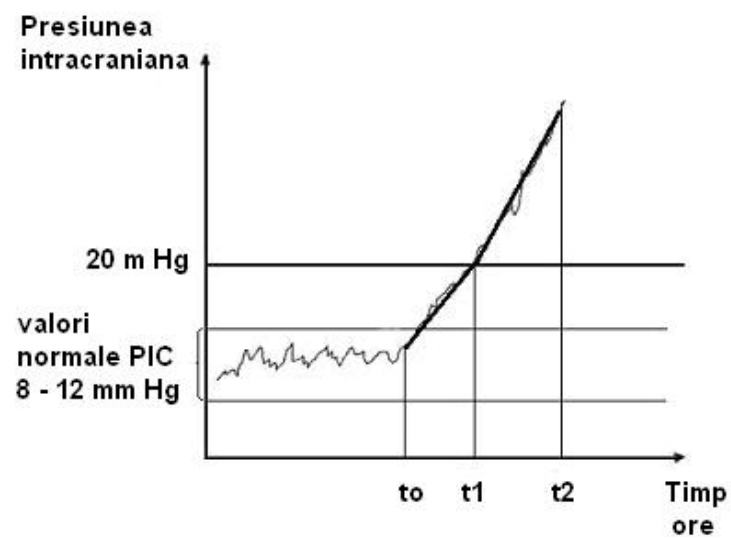

Figure 1

Supra-acute intracranial pressure increase; intervals to-t 1 and $\mathrm{t} 2-\mathrm{t} 1$ are short, of a few hours 
1. the supra-acute intracranial pressure increase with rapid decompensation. The intracranial pressure increase is very rapid up to the value of $20 \mathrm{~mm} \mathrm{Hg}$, and afterwards to a maximum pressure value. This pressure increase occurs in supraacute cases of intracranial hematoma, of traumatic cerebral edema, or in the extending edema of the Sylvian artery malign stroke, when a supplementary volume is rapidly developed at the intracranial level. The infraclinical period is very short and the intracranial hypertension decompensation is accelerated due to the high increasing speed of the supplementary intracranial volume and to the exceeding of the compensating capacities. Pressure increases very rapidly and the pathological values of the intracranial pressure are of up to $30-40 \mathrm{~mm} \mathrm{Hg}$. These pathological values are maintained for a short while due to the rapid decompensation.

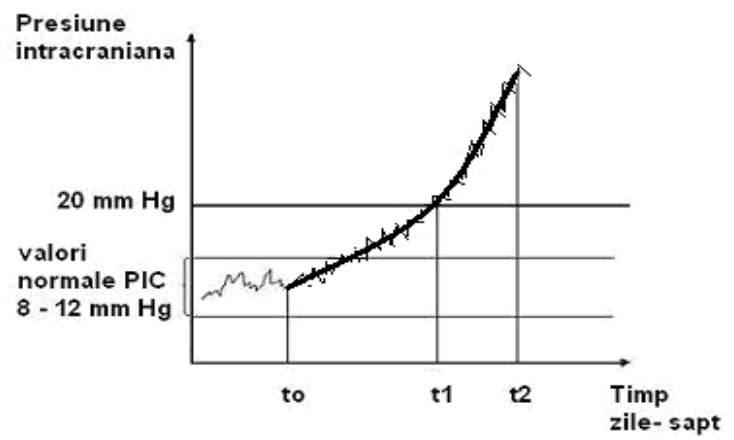

Figure 2

Acute ICP increase, with a prolonged infraclinical period and a rapid decompensation; the interval to-t 1 is prolonged, it may last for weeks or even months, and the decompensation lasts for a few days

2. acute intracranial pressure increase, preceded by a prolonged infraclinical period. In these cases, the period when the intracranial pressure reaches and exceeds the normal pressure values is long, with a slow speed of intracranial pressure increase. This is the type of intracranial pressure increase that occurs in cerebral tumors, cerebral abscesses, chronic subdural hematomas, etc., usually when the occurrence and development of the supplementary intracranial volume is slowly progressive. During the infraclinical period, there is the compensation of the pressure increases caused by the newly added volume, reaching the limit value. Once the compensating capacities have been exceeded, the intracranial pressure increase is rapid up to the maximum values, which are of approximately $30-40 \mathrm{~mm} \mathrm{Hg}$. The period of these pathologic pressure values is short due to the rapid decompensation.

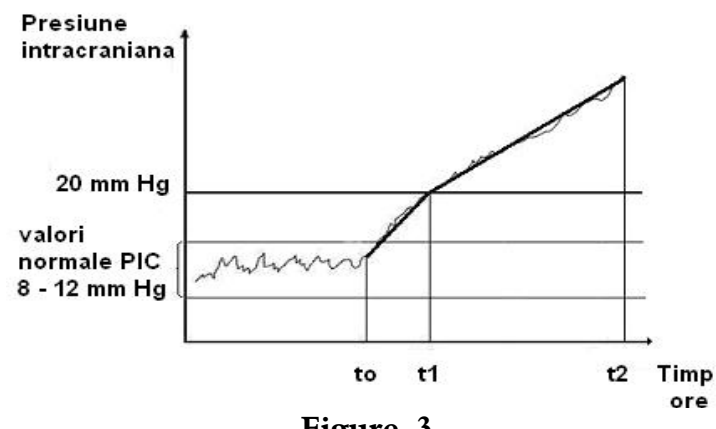

Figure 3

Acute ICP increase, with a short infraclinical period, and a prolonged period of the decompensation risk; the interval to-t1 is short, of a few hours; the interval $\mathrm{t} 1-\mathrm{t} 2$ is prolonged

3. the acute increase, with an infraclinical period of short duration and a longer period of pathological pressure increase. The intracranial pressure increase to the normal limit of $20 \mathrm{~mm} \mathrm{Hg}$ is rapid but slower than the supra-acute pressure increase. Once the normal pressure values have been exceeded, the ICP increase is gradual, but at a slower speed compared to the previous period. It reaches pathological pressure values of approximately $30 \mathrm{~mm} \mathrm{Hg}$. This occurs in hypertensive encephalopathy, which includes a self-limiting mechanism: the intracranial pressure increase caused by the arterial tension increase and the occurrence 
of the cerebral vasodilatation eventually lead to the collabation of the intracranial sanguine vessel walls, with the decrease to a certain degree in the cerebral sanguine volume. It is clinically manifested as an incomplete or complete intracranial hypertension syndrome, which may be decompensated unless appropriately treated. The period when the pathological ICP values are maintained depends on the efficiency of the etio-pathogenic therapy of the arterial hypertension and on the pathogenic therapy of the ICH syndrome.

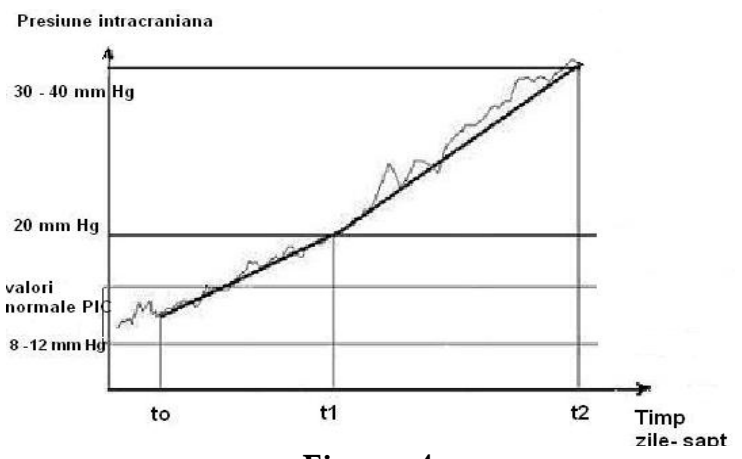

Figure 4

Slow ICP increase, with a long infraclinical period and a prolonged period of pathological pressure values

\section{4. the slow intracranial pressure increase} with a prolonged infraclinical period and a long period of pathological ICP increase. This type of pressure increase occurs in cases of diminished resorption of the cerebrospinal fluid in acute meningitis, subarachnoid hemorrhage, cerebral venous thromboses and thrombosis of dural sinuses, cerebral thrombophlebites, etc. Intracranial pressure increases slowly and gradually until it reaches the normal limit value of $20 \mathrm{~mm} \mathrm{Hg}$, and it continues its slow increase with periods when it returns to normal pressure values, according to the etiology of the ICH syndrome. The compensating capacity of the pressure increase allows a prolonged maintenance of the cerebral sanguine flux, and the period of the pathological pressure values is rather long. There is a development towards a prolonged, complete or incomplete $\mathrm{ICH}$ syndrome, without leading to clinical decompensation.

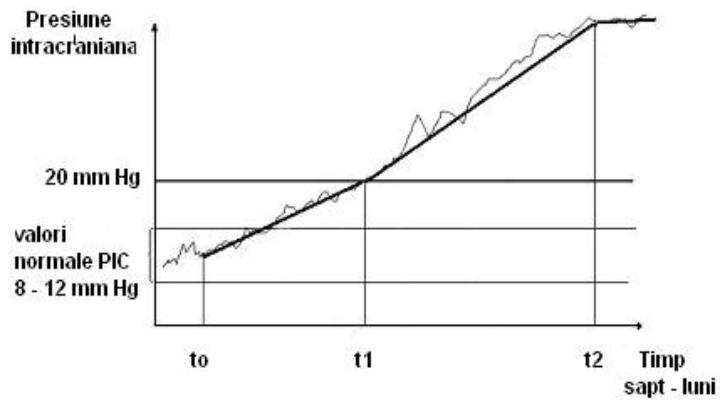

Figure 5

Chronic ICP increase, with prolonged infraclinical period and an extremely long period of pathologic pressure values

5. the "chronic", extremely slow increase in intracranial pressure. This occurs in cases of idiopathic intracranial hypertension and in certain cases of thromboses of intracranial venous sinuses. A very slow intracranial pressure increase occurs, which allows good pressure compensation and the maintenance of the cerebral sanguine flux to almost normal values. The pathogeny of the idiopathic intracranial hypertension syndrome is complex, but the intracranial pressure increase has a "chronic" aspect. The pathogenic ICP values are very high, reaching to $60-80 \mathrm{~mm} \mathrm{Hg}$; these are valued that may be maintained in a plate for long periods of time. There is a clinical evolution towards an incomplete $\mathrm{ICH}$ clinical syndrome, with no decompensation.

Corresponding author:

D. Adam

Neurosurgery, "Carol Davila” University of Medicine and Pharmacy, Bucharest adamdanil950@gmail.com 


\section{References}

1. Lee S, Cho BK, Kim H.Hypertensive encephalopathy with reversible brainstem edema. J Korean Neurosurg Soc. 2013 Aug;54(2):139-41.

2. Suetterlin K, Borg N, Joy H, Lovett JK, Ghosh BC. When is 'idiopathic intracranial hypertension' no longer idiopathic? Pract Neurol. 2013 Oct 29. doi: 10.1136/practneurol-2013-000680.

3. Lindvall P, Koskinen LO. Intracranial Hypertension due to Cerebral Venous Sinus Thrombosis following Head Trauma: A Report of Two Cases. Case Rep Neurol. 2013 Sep 25;5(3):168-74

4. Suh SY, Kim SJ. IIH with normal CSF pressures? Indian J Ophthalmol. 2013 Oct 22.

5. Değerliyurt A, Teber S, Karakaya G, Güven A, Seker ED, Arhan EP, Sayli TR. Pseudotumor cerebri/idiopathic intracranial hypertension in children: An experience of a tertiary care hospital. Brain Dev.
2013 Oct 16. doi:pii: S0387-7604(13)00288-X. 10.1016/j.braindev.2013.09.007.

6. Barnett M, Sinha MD, Morrison D, Lim M Intracranial hypertension presenting with severe visual failure, without concurrent headache, in a child with nephrotic syndrome. BMC Pediatr. 2013 Oct 10;13:167. doi: 10.1186/1471-2431-13-167.

7. Batra R, Sinclair A. Idiopathic intracranial hypertension; research progress and emerging themes. J Neurol. 2013 Oct 2.

8. Aylward SC. Pediatric idiopathic intracranial hypertension: a need for clarification. Pediatr Neurol. 2013 Nov;49(5):303-4. doi: 10.1016/j.pediatrneurol.2013.05.019.

9. Schwartz R, Kliper E, Stern N, Dotan G, Berliner S, Kesler A. The obesity pattern of idiopathic intracranial hypertension in men. Graefes Arch Clin Exp Ophthalmol. 2013 Nov;251(11):2643-6. doi: 10.1007/s00417-013-2420-6. 